\title{
NDM Production as a Dominant Feature in Carbapenem-Resistant Enterobacteriaceae Isolates from a Tertiary Care Hospital
}

\author{
Fakhur Uddin ${ }^{1}$, Syed Hadi Imam ${ }^{2}$, Saeed Khan ${ }^{3}$, Taseer Ahmed Khan ${ }^{4}$, Zulfiqar Ahmed ${ }^{4}$, \\ Muhammad Sohail ${ }^{5, *(\mathbb{D}}$, Ashraf Y. Elnaggar ${ }^{6}(\mathbb{D})$, Ahmed M. Fallatah ${ }^{7}$ and Zeinhom M. El-Bahy ${ }^{8}$
}

check for updates

Citation: Uddin, F.; Imam, S.H.; Khan, S.; Khan, T.A.; Ahmed, Z.; Sohail, M.; Elnaggar, A.Y.; Fallatah, A.M.; El-Bahy, Z.M. NDM Production as a Dominant Feature in

Carbapenem-Resistant Enterobacteriaceae Isolates from a Tertiary Care Hospital. Antibiotics 2022, 11, 48. https://doi.org/ $10.3390 /$ antibiotics 11010048

Academic Editors: Ding-Qiang Chen, Yulong Tan, Ren-You Gan,

Zhenbo Xu, Junyan Liu

and Michele Bartoletti

Received: 18 November 2021

Accepted: 27 December 2021

Published: 31 December 2021

Publisher's Note: MDPI stays neutral with regard to jurisdictional claims in published maps and institutional affiliations.

Copyright: (C) 2021 by the authors. Licensee MDPI, Basel, Switzerland. This article is an open access article distributed under the terms and conditions of the Creative Commons Attribution (CC BY) license (https:// creativecommons.org/licenses/by/ $4.0 /)$.
1 Jinnah Postgraduate Medical Center (JPMC), Department of Microbiology, Basic Medical Sciences Institute (BMSI), Karachi 75510, Pakistan; fakharindhar@hotmail.com

2 Basildon University Hospital Essex, Basildon SS16 5NL, UK; dr_hadiimam@hotmail.com

3 Department of Pathology, Dow University of Health Sciences (DUHS), Karachi 74200, Pakistan; saeed.khan@duhs.edu.pk

4 Department of Physiology, University of Karachi, Karachi 75270, Pakistan; takhan@uok.edu.pk (T.A.K.); zuahmed@uok.edu.pk (Z.A.)

5 Department of Microbiology, University of Karachi, Karachi 75270, Pakistan

6 Department of Food Nutrition Science, College of Science, Taif University, P.O. Box 11099, Taif 21944, Saudi Arabia; aynaggar@tu.edu.sa

7 Department of Chemistry, College of Science, Taif University, P.O. Box 11099, Taif 21944, Saudi Arabia; a.fallatah@tu.edu.sa

8 Department of Chemistry, Faculty of Science, Al-Azhar University, Nasir City, Cairo 11884, Egypt; zeinelbahy@azhar.edu.eg

* Correspondence: msohail@uok.edu.pk

\begin{abstract}
The worldwide spread and increasing prevalence of carbapenem-resistant Enterobacteriaceae (CRE) is of utmost concern and a problem for public health. This resistance is mainly conferred by carbapenemase production. Such strains are a potential source of outbreaks in healthcare settings and are associated with high rates of morbidity and mortality. In this study, we aimed to determine the dominance of NDM-producing Enterobacteriaceae at a teaching hospital in Karachi. A total of 238 Enterobacteriaceae isolates were collected from patients admitted to Jinnah Postgraduate Medical Centre (Unit 4) in Karachi, Pakistan, a tertiary care hospital. Phenotypic and genotypic methods were used for detection of metallo- $\beta$-lactamase. Out of 238 isolates, $52(21.8 \%)$ were CRE and 50 isolates were carbapenemase producers, as determined by the CARBA NP test; two isolates were found negative for carbapenemase production by CARB NP and PCR. Four carbapenemase-producing isolates phenotypically appeared negative for metallo- $\beta$-lactamase (MBL). Of the 52 CRE isolates, $46(88.46 \%)$ were $b l a_{\text {NDM }}$ positive. Most of the NDM producers were Klebsiella pneumoniae, followed by Enterobacter cloacae and Escherichia coli. In all the NDM-positive isolates, the bla $a_{\text {NDM }}$ gene was found on plasmid. These isolates were found negative for the VIM and IPM MBLs. All the CRE and carbapenem-sensitive isolates were sensitive to colistin. It is concluded that the NDM is the main resistance mechanism against carbapenems and is dominant in this region.
\end{abstract}

Keywords: carbapenem-resistant Enterobacteriaceae; metallo- $\beta$-lactamases; NDM producers

\section{Introduction}

Many Enterobacteriaceae species are the pathogens involved in hospital-associated and community-acquired infections, especially in urinary and respiratory tracts, the blood stream, and intra-abdominal and surgical sites [1]. The most commonly encountered pathogens of the family Enterobacteriaceae are Escherichia coli, Klebsiella pneumoniae, Proteus, Salmonella, Shigella and Enterobacter spp. These genera are reportedly very susceptible to carbapenems [2]. Hence, carbapenems are considered as a good option to treat the infections 
caused by extended-spectrum $\beta$-lactamase (ESBL) producing strains and other multidrugresistant (MDR) bacteria [3,4]. Owing to the emergence (up to 32\%) of carbapenem-resistant Enterobacteriaceae (CRE) and their inclusion in the list of priority pathogens, they have received attention globally [4-9]. CRE strains can also spread resistance markers by horizontal transfer to other strains in hospitals and augment problems in healthcare sectors [4]. In the case of bloodstream infections, CRE significantly increase the mortality rate up to $65.4 \%$ in comparison to carbapenemase-susceptible Enterobacteriaceae pathogens [8]. CRE strains have a common mechanism of resistance against carbapenem antibiotics by producing carbapenemases [4].

Many types and subtypes of carbapenemases (bla $a_{\mathrm{IMP}}, b l a_{\mathrm{VIM}}, b l a_{\mathrm{SIM}}, b l a_{\mathrm{SPM}}, b l a_{\mathrm{GIM}}$ $\left.b l a_{\mathrm{KPC}}, b l a_{\mathrm{SME}}\right)$ have been recognized among Enterobacteriaceae; the arrival of NDM-1 is the 'final straw' in this increasing antimicrobial resistance problem [10]. Rapid and easy determination of carbapenemases in Enterobacteriaceae is needed for effective clinical practices and infection control measures. Different phenotypic approaches are employed for the diagnosis of CPE, including the modified Hodge test (MHT), Blue-Carba test and Rapidec CARBA NP test. The Rapidec CARBA NP test is a biochemical assay with a good sensitivity and specificity, but it cannot differentiate among types of carbapenemases [7,11]. This test is also recommended by the Clinical and Laboratory Standards Institute (CLSI) [12]. Likewise, another inhibitory test, EDTA with imipenem or meropenem, is a phenotypic test for the detection of metallo- $\beta$-lactamases in Enterobacteriaceae. Both tests are cost-effective and are feasible for the screening of carbapenemases and metallo- $\beta$-lactamases prior to performing costly and sophisticated genotypic tests in low-income countries.

This cross-sectional, single-center study was conducted on the admitted patients in the medical unit of Jinnah Postgraduate Medical Centre in Karachi, Pakistan. The different clinical specimens were collected according to the site of infection. Traditional microbiological techniques and some cost-effective assays were used for the detection of the carbapenemases, with specific emphasis on metallo- $\beta$-lactamases. The goal of this research was three-fold: to monitor antimicrobial resistance among the members of Enterobacteriaceae, to detect the presence of CRE and to study the prevalence of metallo- $\beta$-lactamases producing Enterobacteriaceae by phenotypic and genotypic methods.

\section{Results}

Among 238 Enterobacteriaceae isolates, E. cloacae $(40 ; 16.80 \%)$, K. pneumonia $(69 ; 28.99 \%)$ and E. coli $(84 ; 35.29 \%)$ were the most common pathogens (Table 1). Klebsiella aerogenes, K. oxytoca, P. mirabilis, P. vulgaris and S. typhi were isolated less frequently. Out of 238 isolates, $52(21.84 \%)$ were CRE. The carbapenem resistance was higher in Klebsiella aerogenes $(3 ; 23.1 \%)$, K. pneumoniae $(20 ; 28.9 \%)$, E. cloacae $(9 ; 22.5 \%)$ and E. coli $(18 ; 21.4 \%)$ in comparison to other species of Enterobacteriaceae (Table 2). The isolates of different species are small in number, and for the validation of these results, the large scale studies are required. The resistance against the ampicillin, cefazolin and cefuroxime was $90-100 \%$. A large number of isolates (46.2\% to $83.3 \%$ ) exhibited resistance against $\beta$-lactamase inhibitors and cephalosporin. The resistance to aminoglycosides was $45-71 \%$ (Figure 1 ), and all the isolates were susceptible to colistin, except the Proteus spp. having intrinsic resistance. Out the 52 CRE isolates, $46(88.5 \%)$ found carbapenemase producers by the phenotypic colorimetric assay, Rapedic CARBA NP. The metallo- $\beta$-lactamase detection was determined by the phenotypic inhibitor based EDTA+ IMP and MEM discs. A total of 43 (82.7\%) showed metallo- $\beta$-lactamase producers using this method. For the confirmation by the PCR assay, $41(78.8 \%)$ were positive for $b l a_{\mathrm{NDM}}$. The $b l a_{\mathrm{VIM}}$ and $b l a_{\mathrm{IMP}}$ could not be detected in any isolate, and out of $52 \mathrm{CRE}, 11(21 \%)$ isolates were negative for $b l a_{\mathrm{NDM}}, b l a_{\mathrm{VIM}}$ and $b l a_{\mathrm{IMP}}$. The $b l a_{\mathrm{NDM}}$ positive isolates were analyzed for the plasmid extraction, and PCR was performed; all the isolates showed the presence of $b l a_{\mathrm{NDM}}$ on plasmids. The conjugation results revealed that $b l a_{\mathrm{NDM}}$ was transferred to $E$. col $\mathrm{J} 53$ recipient successfully from $38 b l a_{\mathrm{NDM}}$ positive isolates, and 03 E. coli isolates failed to show the transmissibility by conjugation on several attempts. 
Table 1. Proportion of different species of Enterobacteriaceae $(n=238)$.

\begin{tabular}{cccc}
\hline Species & Number (\%) of CR & Number (\%) of CS ** & Total No. of Isolates (\%) \\
\hline E. coli & $18(21.42)$ & $66(78.57)$ & $84(35.29)$ \\
K. pneumoniae & $20(28.98)$ & $49(71.01)$ & $69(28.99)$ \\
Enterobacter cloacae & $9(22.50)$ & $31(77.50)$ & $40(16.80)$ \\
Klebsiella aerogenes & $3(23.07)$ & $10(76.92)$ & $13(5.46)$ \\
S. typhi & - & $10(100)$ & $10(4.20)$ \\
P. mirabilis & $2(20.0)$ & $8(80.00)$ & $10(4.20)$ \\
K. oxytoca & - & $6(100)$ & $6(2.52)$ \\
P. vulgaris & - & $4(100)$ & $4(1.68)$ \\
S. marcescens & - & $2(100)$ & $2(0.84)$ \\
Total & $52(21.84)$ & $186(78.15)$ & $238(100)$ \\
\hline
\end{tabular}

${ }^{*} \mathrm{CR}$, carbapenem-resistant isolate (resistant to both imipenem and meropenem); ${ }^{* *} \mathrm{CS}$, carbapenem susceptible.

Table 2. Phenotypic detection of carbapenemases by Rapedic CARBA NP test, MBL detection by EDTA synergy with carbapenems (double disc diffusion test, (DDST)) and carbapenemases by PCR in CRE isolates $(n=52)$.

\begin{tabular}{cccc}
\hline $\begin{array}{c}\text { Carbapenem-Resistant } \\
\text { (CR) Species }\end{array}$ & Rapedic CARBA NP Positive No. (\%), & MBL Positive No. (\%), & $\begin{array}{c}\text { PCR bla } \boldsymbol{a}_{\text {NDM No. (\%), }} \\
\boldsymbol{n}=\mathbf{5 2}\end{array}$ \\
\hline E. coli & $\mathbf{n = 5 2}$ & $15(28.84)$ & $15(28.84)$ \\
K. pneumoniae & $16(30.76)$ & $17(32.69)$ & $16(30.76)$ \\
E. cloacae & $19(36.53)$ & $8(15.38)$ & $7(13.46)$ \\
Klebsiella aerogenes & $8(15.38)$ & $3(5.76)$ & $3(5.76)$ \\
Total No. (\%) & $3(5.76)$ & $43(82.69)$ & $41(78.84)$ \\
\hline
\end{tabular}

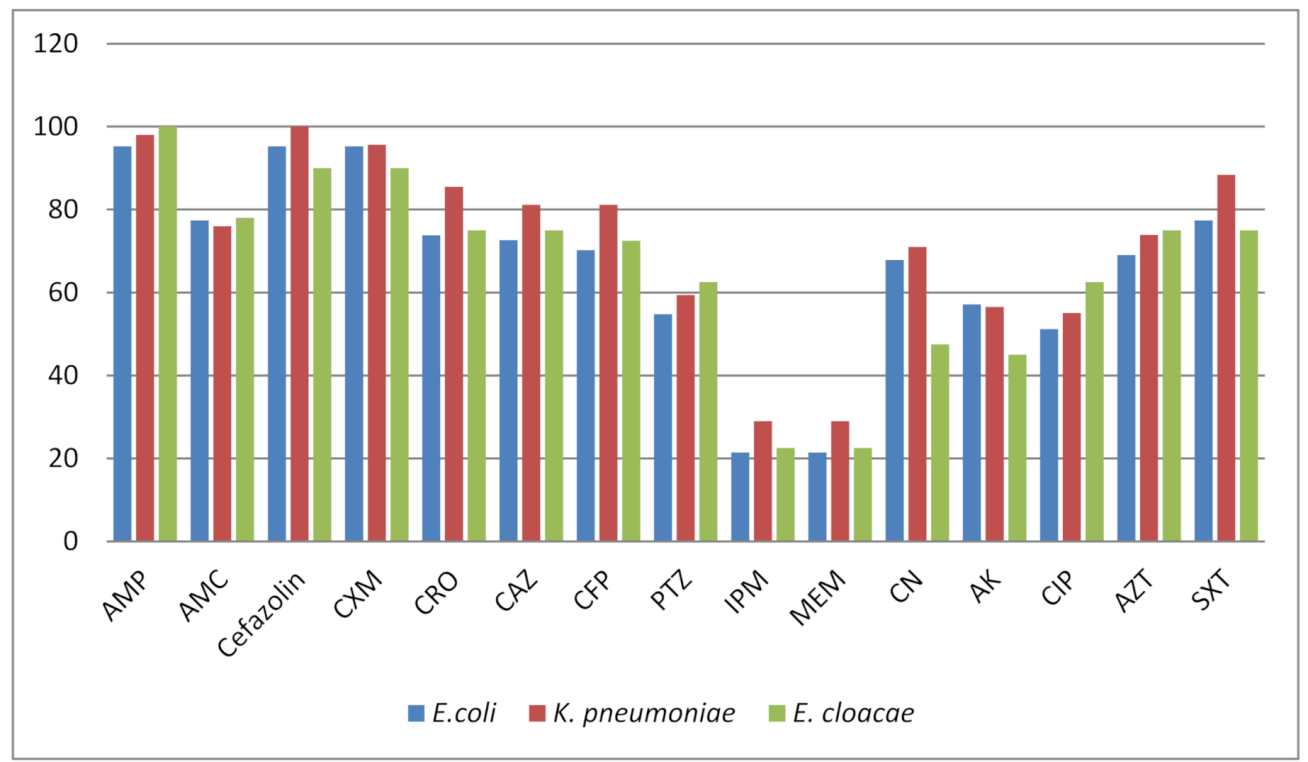

Figure 1. Resistance pattern of major isolated pathogens Escherichia coli, Klebsiella pneumoniae and Enterobacter cloacae. AMP: ampicillin, AMC: amoxicillin/clavulanic acid, CXM: cefuroxime, CRO: ceftriaxone, CAZ: ceftazidime, CFP: Cefepime, PTZ: piperacillin/tazobatam, IPM: imipenem, MEM: meropenem, CN: gentamicin, AK: amikacin, CIP: ciprofloxacin, AZT: aztreonam, SXT: sulfamethoxazole-trimethoprim.

\section{Discussion}

The incidence of infection by the carbapenem-resistant Enterobacteriaceae (CRE) is increasing worldwide and poses a threat to public health and a challenge for physicians [2]. Among CREs, K. pneumoniae is a leading pathogen, followed by E. coli and E. cloacae [13]. The common mode for carbapenem resistance in Enterobacteriaceae is the production of 
carbapenemases, particularly New Delhi metallo- $\beta$-lactamase (NDM-1), on the Indian subcontinent [14]. The carbapenem resistance and carbapenemase production in Enterobacteriaceae vary geographically with respect to prevalence, pathogen and type of carbapenemase, so it is necessary to continuously monitor the presence and prevalence of these bugs [15]. Although, K. pneumoniae Carbapenemase-2 (KPC-2) has been found dominantly in China, NDM is the most frequently detected $(71.4 \%)$ metallo- $\beta$-lactamase in Hunan among CRE isolates, as reported by Chinese network for CRE surveillance [13]. The prevalence of carbapenem resistance in Enterobacteriaceae isolates was $21.84 \%$ in the present study. The resistance to other classes of $\beta$-lactams, including cephalosporins, penicillins and aztreonam, was higher (61-100\%) in comparison to ciprofloxacin and aminoglycosides. This higher resistance to penicillins and cephalosporins may be attributed to the higher prevalence (60-80\%) of ESBLs, modification in the outer membrane porins or AmpC overexpression or other mechanisms as deciphered for the isolates from Asia [16].

Comparing carbapenem resistance among the members of Enterobacteriaceae, most of the K. pneumoniae isolates appeared resistant, followed by Enterobacter spp. and E. coli. In the majority of CRE isolates $(88.5 \%)$, carbapenemase production was the main mode of resistance to carbapenems by the phenotypic Rapidec CARBA NP test. This is a colorimetric, phenotypic test that is easy to perform without any special requirements. A simple color change can be read by the technician, so it does not require highly skilled or specially trained staff. This provides rapid identification of carbapenemase-producing strains within $30 \mathrm{~min}$ to $2 \mathrm{~h}$, at a lower cost in comparison to the molecular assays [17]. This is helpful for the screening of carbapenemase-producing isolates, especially in low- and middle-income countries, including Pakistan, where molecular assays are not very common. The prevalence of metallo- $\beta$-lactamases was higher $(82.69 \%)$ in CRE by phenotypic assay in the present study. These results were in accordance with the PCR results; therefore, this cost-effective technique may be used to screen metallo- $\beta$-lactamases in CRE isolates and provide information regarding the selection of therapeutic options.

NDM production is the main mode of resistance against the carbapenems in enterobactericeae, as found in this study. NDM was initially reported in K. pneumoniae and E. coli strains isolated from a Swedish patient, having history of seeking medical care in New Delhi, India, in 2009. It has since been spread all over the world and has been detected in different species of Enterobacteriaceae as well as in other Gram-negative bacilli. Unlike in North America and Europe, NDM, IMP and VIM are the most common carbapenemases in CRE in Southeast Asia and were similarly observed in the present work in Karachi, Pakistan. The previous data revealed that the NDM is endemic in Pakistan, Bangladesh and India, while KPCs are endemic in Colombia, Brazil, Argentina and USA [7,13]. Surveillance reports from India and neighboring countries summarized that, in Enterobacteriaceae, the most predominant carbapenemase is NDM [7]. These findings coincide with the present work. The higher prevalence of NDM producers in this study revealed that the NDMharboring isolates are dominant in this region. A previous study from Pakistan reported that the major carbapenemase among the carbapenemase-producing Enterobacteriaceae is NDM [18].

The results of the plasmid DNA extraction and conjugation assay indicated that the $b l a_{\mathrm{NDM}}$ is plasmid mediated, although a more sensitive assay needs to be performed to confirm this conclusion. Similar findings were reported in an earlier report [15]. In the present research work, the $b l a_{\mathrm{VIM}}$ and $b l a_{\mathrm{IPM}}$ could not be detected in the CRE isolates. However, these are reported in the members of Enterobacteriaceae isolates in the regions and health care settings where $b l a_{\mathrm{VIM}}$ and $b l a_{\mathrm{IPM}}$ carrying $P$. aeruginosa and other glucose non-fermenter Gram-negative bacilli are common $[19,20]$. In the present work six CRE isolates were negative for carbapenemase production by the Rapidec CARBA NP test, and 11 isolates were negative for $b l a_{\mathrm{NDM}}, b l a_{\mathrm{IPM}}$ and $b l a_{\mathrm{VIM}}$ by PCR. These isolates may carry any AmpC, KPC or OXA carbapenemses in combination with other carbapenem-resistant mechanisms, including overexpression of efflux pumps and the decreased permeability to carbapenems. 


\section{Materials and Methods}

\subsection{Setting}

The study was conducted in the Department of Microbiology, Basic Medical Sciences Institute (BMSI) and Medical Unit-4 of Jinnah Postgraduate Medical Centre (JPMC) Karachi, Pakistan, in collaboration with the department of Microbiology and the department of Physiology, University of Karachi.

\subsection{Sample Size and Collection}

A total of 238 Enterobacteriaceae isolates were included in this study. The clinical specimens were collected from January to June 2018 from medical Unit- 4 (Department of Medicine and Critical Care), which comprises a general ward and an ICU. The samples were collected from the urine patients suspecting of having urinary tract infections, from respiratory secretions including endotracheal tubes, tracheal aspirates and sputum for respiratory tract infections, and from blood in the case of septicemia. One isolate per patient was included, and the repeated samples of the same patient were excluded in the present study according to the adjusted criteria.

Inclusion criteria: During this period all the admitted patients presenting urinary tract infection, hospital acquired pneumonia and bacteremia were included.

Exclusion criteria: Isolates other than Enterobacteriaceae and growth-negative specimens were excluded.

\subsection{Identification of the Isolates}

Bacterial isolates were identified by the routine techniques, which comprised cultural and morphological features and a battery of biochemical and motility tests. The species identification was further confirmed by API 20E (BioMérieux, Lyon, France). The antimicrobial susceptibility testing (AST) was determined by the disc diffusion technique following the CLSI recommendations and protocol [12]. Colistin susceptibility testing was performed by the broth microdilution method [12]. The quality control strains included E. coli (ATCC 25922) for AST and Pseudomonas aeruginosa (ATCC 27853) for carbapenem resistance. The break points for imipenem (IPM) and meropenem (MEM) against Enterobacteriaceae were interpreted as sensitive, intermediate and resistant at the MICs

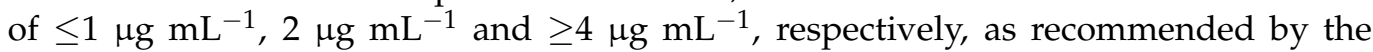
CLSI [12]. The MICs were performed by the Etest strip (BioMérieux, Lyon, France). The Rapedic CARBA NP (RCNP) kit was used for the phenotypic detection of carbapenemases. The test was performed according to the manufacturer's instructions and standard operating procedures (BioMérieux, Lyon, France). The metallo- $\beta$-lactamases were phenotypically screened and detected by the double disc diffusion method using EDTA as a metallo- $\beta$ lactamase inhibitor and imipenem (IPM, $10 \mu \mathrm{g}$ ) and meropenem (MEM, $10 \mu \mathrm{g})$ disc, (Oxoid, Basingstoke, Hampshire, UK). After lawn formation on Muller Hinton agar (MHA) surface, IPM and MEM discs were placed $30 \mathrm{~mm}$ apart from one another, and a filter paper disc having $10 \mu \mathrm{L}$ of $0.5 \mathrm{M}$ EDTA solution was placed at the center of both discs. Inoculated plates were incubated at $37^{\circ} \mathrm{C}$ overnight [21]. The synergistic effect of EDTA and carbapenems against metallo- $\beta$-lactamase producers appeared when the zone of inhibition due to the carbapenem discs increased with EDTA-containing discs.

\subsection{Manual PCR Method}

The RCNP positive isolates were selected to analyze metallo- $\beta$-lactamase production by targeting common genes, including $b l a_{V I M}$ (F-GGTGTTTGGTCGCATATCGC RCCATTCAGCCAGATCGGCATC), $b l a_{N D M}$ (F-CACCTCATGTTTGAATTCGCC R-CTCTGT CACATCGAAATCGC) and $b l a_{I P M}$ (F-GGAATAGAGTGGCTTAATTC R-CAACCAGTTTTG CCTTACC), with 503bp, 984bp and 327bp, respectively. PCR reaction $(25 \mu \mathrm{L})$ was prepared in according to the master mix protocol (Promega, Madison, WI, USA). The PCR conditions were maintained as previously described $[19,22]$. 


\subsection{Plasmid Extraction and Conjugation}

The GeneJET plasmid miniprep kit (Thermo Scientific ${ }^{\mathrm{TM}}$, Waltham, MA, USA, \#K0502) was used for the plasmid extraction, according to the manufacturer's instructions and the protocol for amplification of $b l a_{V I M}, b l a_{I M P}$ and $b l a_{N D M}$. The PCR mixture and conditions were the same as for the whole DNA sample amplification. Conjugation was performed as described by Borgia et al. [23]. The PCR positive $b l a_{N D M}$ CRE strains were used as the donors with the recipient E. coli J53 (a sodium azide resistant) strain. The transconjugants were selected by inoculating a mixture of donor and recipient fresh cultures on MuellerHinton $(\mathrm{MH})$ agar containing sodium azide $\left(100 \mu \mathrm{g} \mathrm{mL}^{-1}\right)$ and imipenem $\left(1 \mu \mathrm{g} \mathrm{mL}{ }^{-1}\right)$. The $b l a_{N D M}$ gene was detected in transconjugants by using PCR, with the same primers and conditions used for the whole genome DNA and plasmid of clinical isolates.

\section{Conclusions}

In conclusion, this study supports the dominance of NDM in this setting and supports continuous monitoring to control outbreaks and infection mitigating measures against the spread of these bugs.

Author Contributions: Conceptualization, F.U., M.S., S.H.I., S.K. and A.Y.E.; methodology, F.U., M.S., A.M.F., T.A.K. and Z.A.; software, Z.M.E.-B. and S.H.I.; validation, M.S., A.Y.E., S.K., Z.A. and A.M.F.; formal analysis, A.M.F., T.A.K. and Z.A.; investigation, F.U., M.S., T.A.K. and Z.A.; resources, A.M.F., Z.M.E.-B. and S.H.I.; data curation, F.U., M.S. and S.H.I.; writing-original draft preparation, F.U. and M.S.; writing—review and editing, all authors; supervision, M.S., S.H.I. and S.K.; project administration, A.Y.E., A.M.F. and Z.M.E.-B.; funding acquisition, A.Y.E., A.M.F. and Z.M.E.-B. All authors have read and agreed to the published version of the manuscript.

Funding: This research was funded by Taif research Supporting Project (TURSP-2020/46) Taif University Taif, Saudi Arabia.

Institutional Review Board Statement: Informed consent was obtained from the patient or attendant, and ethical approval was received from the institutional review board (IRB), JPMC, Karachi (No. F.2-18/2014-GENL/31649577/JPMC). This work was the extended part of research conducted on "MBLs in Pseudomonas and Acinetobacter".

Informed Consent Statement: Informed consent was obtained from all subjects involved in the study.

Data Availability Statement: Data associated with this work are given in the manuscript. Raw data can be obtained from the corresponding author upon a reasonable request.

Acknowledgments: The authors acknowledge Taif University Research Supporting Project (TURSP2020/46) Taif University Taif, Saudi Arabia.

Conflicts of Interest: The authors declare no conflict of interest.

\section{References}

1. Wong, M.H.; Chan, E.W.; Chen, S. Isolation of carbapenem-resistant Pseudomonas spp. from food. J. Glob. Antimicrob. Resist. 2015, 5, 109-114. [CrossRef]

2. Doi, Y. Treatment options for carbapenem-resistant gram-negative bacterial infections treatment options for carbapenem-resistant gram-negative bacterial infections. Clin. Infect. Dis. 2018, 69, S565-S575. [CrossRef] [PubMed]

3. Marco, C.; Alberto, A.; Tommaso, G.; Teresa, S.; Marzia, L.F.; Carla, F.; Mirandola, W.; Gargiulo, R.; Barozzi, A.; Mauri, C.; et al. Multicenter evaluation of the RAPIDEC! CARBA NP test for rapid screening of carbapenemase-producing Enterobacteriaceae and Gram-negative nonfermenters from clinical specimens. Diagn. Microbiol. Infect. Dis. 2017, 88, 207-213. [CrossRef]

4. Nordmann, P.; Poirel, L.; Walsh, T.R.; Livermore, D.M. The emerging NDM carbapenemases. Trends. Microbiol. 2011, 19, 588-595. [CrossRef]

5. Rossolini, G.M. Extensively drug-resistant carbapenemase-producing Enterobacteriaceae: An emerging challenge for clinicians and healthcare systems. J. Intern. Med. 2015, 277, 528-531. [CrossRef] [PubMed]

6. Suwantarat, N.; Carroll, K.C. Epidemiology and molecular characterization of multidrug-resistant Gram-negative bacteria in Southeast Asia. Antimicrob. Resist. Infect. Control 2016, 4, 15. [CrossRef]

7. Nordmann, P.; Poirel, L. Epidemiology and diagnostics of carbapenem resistance in gram-negative bacteria. Clin. Infect. Dis. 2019, 69, S521-S528. [CrossRef] 
8. Logan, L.K.; Weinstein, R.A. The Epidemiology of carbapenem-resistant Enterobacteriaceae: The impact and evolution of a global menace. J. Infect. Dis. 2017, 215, S28-S36. [CrossRef]

9. Li, X.; Ye, H. Clinical and mortality risk factors in bloodstream infections with carbapenem-resistant Enterobacteriaceae. Can. J. Infect. Dis. Med. Microbiol. 2017, 6212910. [CrossRef]

10. Mitra, S.; Mukherjee, S.; Naha, S.; Chattopadhyay, P.; Dutta, S.; Basu, S. Evaluation of co-transfer of plasmid-mediated fluoroquinolone resistance genes and bla $a_{N D M}$ gene in Enterobacteriaceae causing neonatal septicaemia. Antimicrob. Resist. Infect. Control 2019, 8. [CrossRef]

11. Rao, M.R.; Chandrashaker, P.; Mahale, R.P.; Shivappa, S.G.; Gowda, R.S.; Chitharagi, V.B. Detection of carbapenemase production in Enterobacteriaceae and Pseudomonas species by carbapenemase Nordmann-Poirel test. J. Lab. Physicians 2019, 11, 107-110. [CrossRef]

12. Clinical \& Laboratory Standards Institute (CLSI). Performance Standards for Antimicrobial Susceptibility Testing; 28th Informational Supplement. M100-S28; CLSI: Wayne, PA, USA, 2018.

13. Zhang, Y.; Wang, Q.; Yin, Y.; Chen, H.; Jin, L.; Gu, B.; Xie, L.; Yang, C.; Ma, X.; Li, H.; et al. Epidemiology of carbapenem-resistant Enterobacteriaceae infections: Report from the China CRE Network. Antimicrob. Agents Chemother. 2018, 62, e01882-17. [CrossRef]

14. Dortet, L.; Poirel, L.; Nordmann, P. Worldwide dissemination of the NDM-type carbapenemases in Gram-negative bacteria. BioMed Res. Int. 2014, 2014, 249856. [CrossRef]

15. Yang, Q.; Fang, L.; Fu, Y.; Du, X.; Shen, Y.; Yu, Y. Dissemination of NDM-1-producing Enterobacteriaceae mediated by the IncX3-type plasmid. PLoS ONE 2012, 5, e0129454. [CrossRef]

16. Abrar, S.; Hussain, S.; Khan, R.A.; Ul-Ain, N.; Haider, H.; Riaz, S. Prevalence of extended-spectrum- $\beta$-lactamase-producing Enterobacteriaceae: First systematic meta-analysis report from Pakistan. Antimicrob. Resist. Infect. Control 2018, 7, 26. [CrossRef]

17. Garg, A.; Garg, J.; Upadhyay, G.C.; Agarwal, A.; Bhattacharjee, A. Evaluation of the Rapidec Carba NP test kit for detection of carbapenemase-producing Gram-Negative Bacteria. Antimicrob. Agents Chemother. 2015, 59, 7870-7872. [CrossRef]

18. Masseron, A.; Poirel, L.; Ali, B.J.; Syed, M.A.; Nordmann, P. Molecular characterization of multidrug-resistance in Gram-negative bacteria from the Peshawar teaching hospital, Pakistan. New Microbes New Infect. 2019, 32, 100605. [CrossRef]

19. Kaase, M.; Nordmann, P.; Wichelhaus, T.A.; Gatermann, S.G.; Bonnin, R.A.; Poirel, L. NDM-2 carbapenemase in Acinetobacter baumannii from Egypt. J. Antimicrob. Chemother. 2011, 66, 1260-1262. [CrossRef]

20. Manenzhe, R.I.; Zar, H.J.; Nicol, M.P.; Kaba, M. The spread of carbapenemase-producing bacteria in Africa: A systematic review. J. Antimicrob. Chemother. 2015, 70, 23-40. [CrossRef]

21. Pandya, N.P.; Prajapati, S.B.; Mehta, S.J.; Kikani, K.M.; Joshi, P.J. Evaluation of various methods for detection of metallo- $\beta$ lactamase (MBL) production in gram-negative bacilli. Int. J. Biol. Med. Res. 2011, 2, 775-777.

22. Wolter, D.J.; Khalaf, N.; Robledo, I.E.; Vázquez, G.J.; Santé, M.I.; Aquino, E.E.; Goering, R.V.; Hanson, N.D. Surveillance of carbapenem-resistant Pseudomonas aeruginosa isolates from Puerto Rican Medical Center Hospitals: Dissemination of KPC and IMP-18 B-lactamases. Antimicrob. Agents Chemother. 2009, 53, 1660-1664. [CrossRef]

23. Borgia, S.; Lastovetska, O.; Richardson, D.; Eshaghi, A.; Xiong, J.; Chung, C.; Baqi, M.; McGeer, A.; Ricci, G.; Sawicki, R.; et al. Outbreak of carbapenem-resistant Enterobacteriaceae containing blaNDM-1, Ontario, Canada. Clin. Infect. Dis. 2012, 55, e109-e117. [CrossRef] 